\title{
IS RESECTION OF PULMONARY AND HEPATIC METASTASES WARRANTED IN PATIENTS WITH COLORECTAL CANCER?
}

Brigitta J. Robinson, MDa

Thomas W. Rice, MDa

Scott A. Strong, MD $^{\mathrm{b}}$

Lisa A. Rybicki, MS

Eugene H. Blackstone, MD ${ }^{\mathrm{a}, \mathrm{c}}$
Background: Conventional management of stage IV colorectal carcinoma is palliative. The value of resecting both liver and lung colorectal metastases that occur in isolation of other sites of metastasis is undetermined. Objectives: Our objectives were to (1) assess the efficacy of resecting both hepatic and pulmonary metastases, (2) investigate the influence of the sequence and timing of metastases, and (3) identify the profile of patients likely to benefit from both hepatic and pulmonary metastasectomy. Patients and methods: Of 48 patients identified with resection of colorectal cancer and, at some point in time, both liver and lung metastases, 25 patients underwent metastasectomy (resection group). The remaining 23 patients comprised the nonresection group. Risk factors for death were identified by multivariable analyses. Results: Median survival was longer after the last metastatic appearance in the resection group (16 months) than in the nonresection group ( 6 months; $P<.001)$. The pattern of risk also differed; it peaked at 2 years and then declined in the resection group but was constant in the nonresection group. In the resection group, patients with metachronous resections survived longer after colorectal resection (median, 70 months) than patients with synchronous (median, 22 months) or mixed resections (median, 31 months; $P<.001$ ). Risk factors for death included older age, multiple liver metastases, and a short disease-free interval. Conclusions: Younger patients with solitary metachronous metastases to the liver, then the lung, and long disease-free intervals are more likely to benefit from resection of both liver and lung metastases. Patients with risk factors also had better survival with resection than without resection. (J Thorac Cardiovasc Surg 1999;117:66-76)
C onventional management of stage IV colorectal carcinoma in most patients is palliative. However, chemotherapy, the mainstay in the treatment of systemic disease, seldom produces long-term survival. In an occasional patient with metastatic disease that is confined to the liver or lung, metastasectomy may be curative.

Metastasectomy in some patients with colorectal metastases to both liver and lung has been reported. ${ }^{1-7}$

From the Departments of Thoracic and Cardiovascular Surgery, Colorectal Surgery, ${ }^{\mathrm{b}}$ and Biostatistics and Epidemiology, ${ }^{\mathrm{c}}$ The Cleveland Clinic Foundation, Cleveland, Ohio.

Read at the Seventy-eighth Annual Meeting of The American Association for Thoracic Surgery, Boston, Mass, May 3-6, 1998.

Received for publication May 8, 1998; revisions requested July 8, 1998; revisions received Sept 10, 1998; accepted for publication Sept 14, 1998.

Address for reprints: Thomas W. Rice, MD, The Cleveland Clinic Foundation, Desk F25, 9500 Euclid Ave, Cleveland, OH 44195.

Copyright (C) 1999 by Mosby, Inc.

$0022-5223 / 99 \$ 8.00+0 \quad \mathbf{1 2 / 6 / 9 4 4 9 1}$
However, identifying candidates for this aggressive surgical approach is controversial because the tumor and host factors that allow systemic disease to be controlled with local therapy are unknown. Therefore the purposes of this study were (1) to evaluate the efficacy of resection of both hepatic and pulmonary colorectal metastases, (2) to investigate the influence of the sequence and timing of metastases, and (3) to identify the profile of patients most likely to benefit from both hepatic and pulmonary metastasectomies.

\section{Patients and methods}

Patient groups. Forty-eight patients at The Cleveland Clinic Foundation with resected colorectal carcinoma and only hepatic and pulmonary metastases were identified. During this time period, 5787 patients had resections of primary colorectal carcinomas; 466 patients had resections of isolated colorectal hepatic metastases; and 72 patients had resections of isolated pulmonary metastases. The selection of patients for resection was not by formal protocol but by clinical evaluation. 


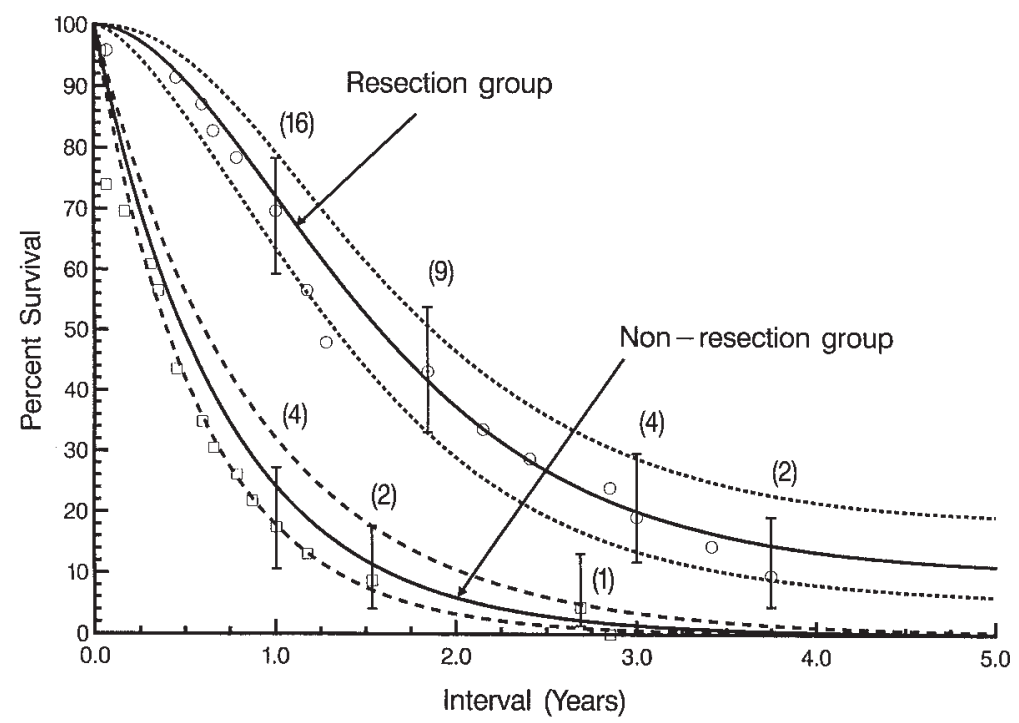

Fig 1. Survival after the last appearance or resection of metastases in the resection and nonresection groups. Each symbol represents a death, positioned according to Kaplan-Meier nonparametric estimates with vertical bars equivalent to $1 \mathrm{SE}$. The solid lines are parametric survival estimates enclosed within confidence intervals (dashed lines) equivalent to $1 \mathrm{SE}$. The numbers of patients living and being followed up are shown in parentheses.

Resection group. From the prospective pathologic database and subsequent review of medical records of patients undergoing resection of colorectal carcinoma between March 1979 and March 1998, 25 patients were identified who had had resection of both hepatic and pulmonary metastasectomies and had no evidence at that time of metastases to other organs.

Nonresection group. From the prospective tumor registry begun in 1986 and subsequent review of medical records, 23 patients were identified through 1996 with resected colorectal carcinoma who had both hepatic and pulmonary metastases, had not undergone both hepatic and pulmonary metastasectomies, and had no evidence at the time of metastases to other organs.

Comparison of groups. Categoric variables within each group were summarized by number and percent of patients in each category. Continuous variables within each group were summarized by mean, standard deviation, median, minimum, and maximum. Differences between the resection and nonresection groups were compared with the $\chi^{2}$ test for categoric variables and the $t$ test for continuous variables, adjusted, when appropriate, for unequal variances.

\section{Benefits of metastasectomy}

End point. The end point for the assessment of efficacy was death. Vital status was available in the respective databases. For patients alive when their medical records were retrieved, telephone interviews were conducted to update vital status. No patient was lost to follow-up.

Comparison of survival. Two versions of follow-up were used in the analysis of survival: (1) from colorectal resection to last follow-up (first cancer resection) and (2) from last
Table I. Timing and sequence of pulmonary and hepatic metastases in relation to colorectal cancer resection

\begin{tabular}{|c|c|c|c|c|}
\hline \multirow{2}{*}{$\begin{array}{l}\text { Timing and sequence } \\
\text { of metastases }\end{array}$} & \multicolumn{2}{|c|}{$\begin{array}{c}\text { Resection } \\
(n=25)\end{array}$} & \multicolumn{2}{|c|}{$\begin{array}{c}\text { Nonresection } \\
(n=23)\end{array}$} \\
\hline & $n$ & $\%$ & $n$ & $\%$ \\
\hline Synchronous* & 2 & 8 & 21 & 91 \\
\hline Metachronous & 17 & 68 & 0 & 0 \\
\hline Colon $\rightarrow$ liver $\rightarrow$ lung & 12 & 48 & 0 & 0 \\
\hline Colon $\rightarrow$ lung $\rightarrow$ liver & 5 & 20 & 0 & 0 \\
\hline Mixed & 6 & 24 & 2 & 9 \\
\hline Colon and lung $\rightarrow$ liver & 1 & 4 & 1 & 4 \\
\hline Colon and liver $\rightarrow$ lung & 5 & 20 & 1 & 4 \\
\hline
\end{tabular}

resection or appearance of metastases to last follow-up (last metastatic appearance). The latter is the time at which all patients were first known to have metastases to both liver and lung.

For both versions, nonparametric estimates of survival were obtained by the Kaplan-Meier method, separately for the resection group, the nonresection group, and the combined groups. ${ }^{8}$ Univariable comparisons between the groups were evaluated by the log-rank test. For the second version, a parametric method was used to resolve the number of hazard phases, identify the shape of the hazard function, and estimate its parameters. ${ }^{9}$ 


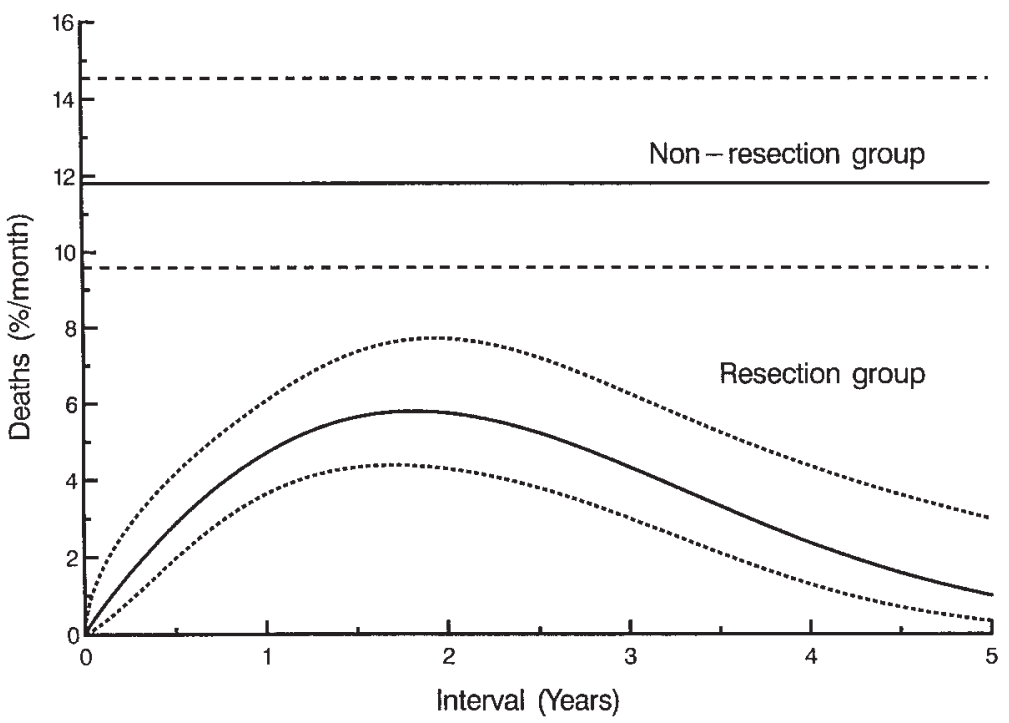

Fig 2. Instantaneous risk of death (hazard function) after last appearance or resection of metastases for resection and nonresection groups.

Table II. Intervals between colorectal cancer resection and appearance or resection of metastases

\begin{tabular}{|c|c|c|c|c|c|c|c|}
\hline & \multirow[b]{2}{*}{ Group } & \multicolumn{5}{|c|}{ Timing (mo) } & \multirow[b]{2}{*}{$\mathrm{P}$ value* } \\
\hline & & $N$ & Mean & $S D$ & Median & Range & \\
\hline \multicolumn{8}{|l|}{ All cases } \\
\hline \multirow{2}{*}{ Colorectal to liver metastasis } & Resection & 25 & 17.0 & 17.6 & 9 & $0-66$ & $<.001$ \\
\hline & Nonresection & 23 & 0.8 & 2.9 & 0 & $0-13$ & -† \\
\hline \multirow[t]{2}{*}{ Colorectal to lung metastasis } & Resection & 25 & 27.2 & 23.4 & 18 & $1-88$ & $<.001$ \\
\hline & Nonresection & 23 & 0.6 & 1.7 & 0 & $0-8$ & $-\dagger$ \\
\hline \multicolumn{8}{|l|}{ Metachronous cases } \\
\hline \multirow[t]{2}{*}{ Colorectal cancer to first metastasis } & Resection & 17 & 19.6 & 12.1 & 18 & $4-43$ & - \\
\hline & Nonresection & 0 & - & - & - & - & \\
\hline \multirow[t]{2}{*}{ Colorectal cancer to second metastasis } & Resection & 17 & 39.1 & 23.4 & 37 & $7-88$ & - \\
\hline & Nonresection & 0 & - & - & - & - & \\
\hline \multirow[t]{2}{*}{ Second metastasis to third metastasis } & Resection & 17 & 19.5 & 16.5 & 13 & $1-53$ & - \\
\hline & Nonresection & 0 & - & - & - & - & \\
\hline \multicolumn{8}{|l|}{ Mixed cases } \\
\hline \multirow[t]{2}{*}{ Mixed metastases to second metastasis } & Resection & 6 & 16.6 & 14.9 & 11 & $5-44$ & .6 \\
\hline & Nonresection & 2 & 10.8 & 4.1 & 11 & $8-14$ & \\
\hline
\end{tabular}

*t Test.

$\dagger$ Cochran and Cox adjustment to the $t$ test (unequal variances).

\section{Timing and sequence of metastases}

Timing. Appearance of hepatic and pulmonary metastases was defined as either synchronous (both hepatic and pulmonary metastases identified within 3 months of colon resection), metachronous (the primary disease and each metastatic site identified separately with more than 3 months between each of the 3 appearances), or mixed (the primary and one metastatic site identified synchronously and the second metastatic site appeared more than 3 months later).

Influence of timing. The influence of timing of the appearance of metastases was assessed in 2 ways. First, survival after the first cancer resection was used to assess whether longevity was different for synchronous, mixed, or metachronous metastases. However, survival assessed in this fashion is potentially biased upward because patients with nonsynchronous metastases must survive long enough for the sequence of metastases to become manifest. Therefore when the second version of survival was used (time 0 being the time when all metastases were diagnosed), variables were entered into the analysis to account for the intervals between the colorectal cancer resection and the appearance of the metastases.

Influence of sequence. In the multivariable analyses, from 


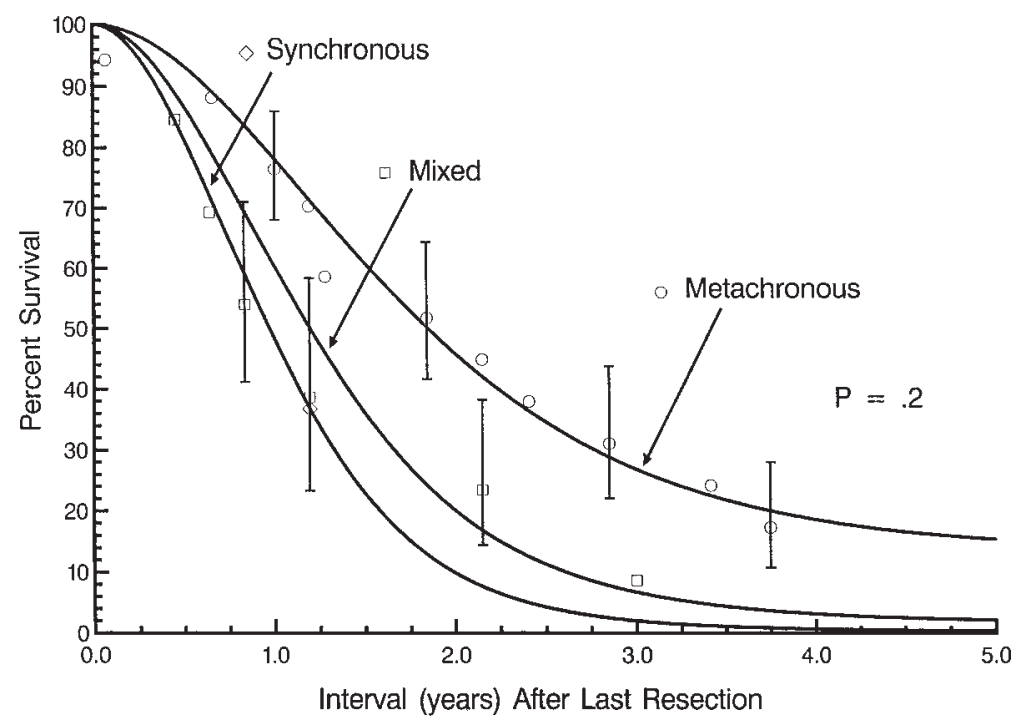

Fig 3. Survival in the resection group after the last resection of the metastases, stratified according to the timing of the metastases: synchronous, metachronous, or mixed.

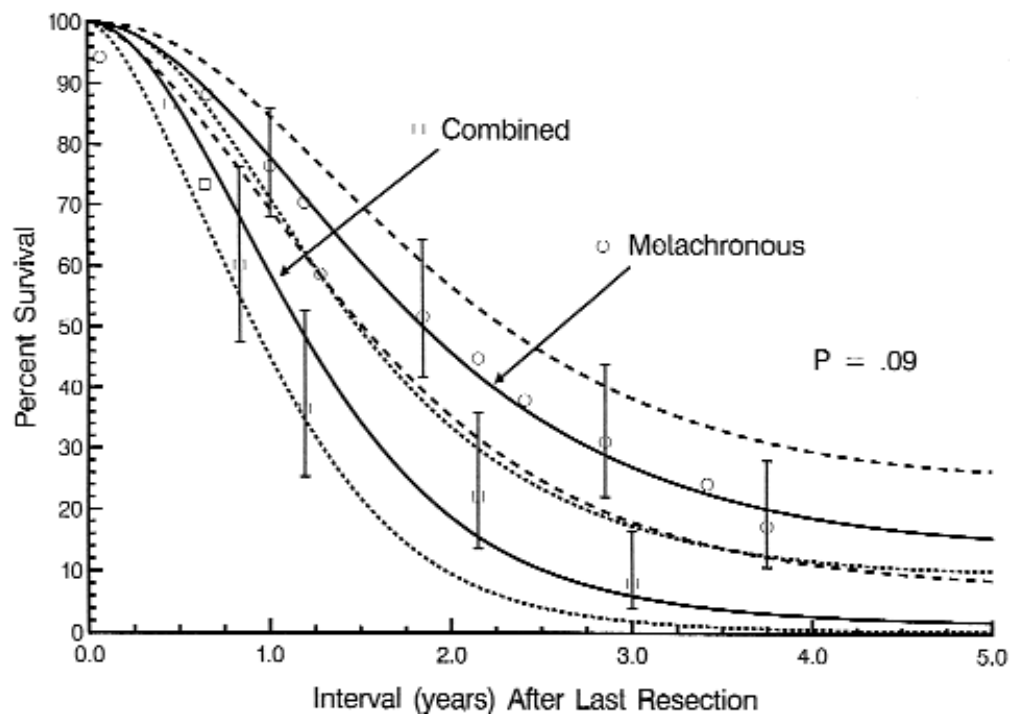

Fig 4. Survival in the resection group as in Fig 3, except the synchronous and mixed subgroups are combined.

the time of last appearance of metastases, variables were assessed that identified the order in which metastases appeared (both together, liver then lung, lung then liver).

The ideal patient for metastasectomy. Patient characteristics that increased or decreased the risk of death were identified by multivariable analyses.

Study variables. The following variables were entered into the multivariable analyses: age, gender, site of colorectal primary (right, a primary carcinoma originating proximal to the midtransverse colon; left, a primary carcinoma originating distal to the midtransverse colon including the rec- tum), TNM stage of the primary colorectal carcinoma, date of colorectal resection, adjuvant therapy after colorectal resection, sequence and timing of metastases, number of hepatic metastases, date of appearance or resection of hepatic metastases, number of pulmonary metastases, unilateral or bilateral pulmonary metastases, date of appearance or resection of pulmonary metastases, follow-up date, and vital status.

Multivariable analyses

General CONDUCt of the multivariable anAlyses. Exploratory analysis included correlation analysis, stratified 


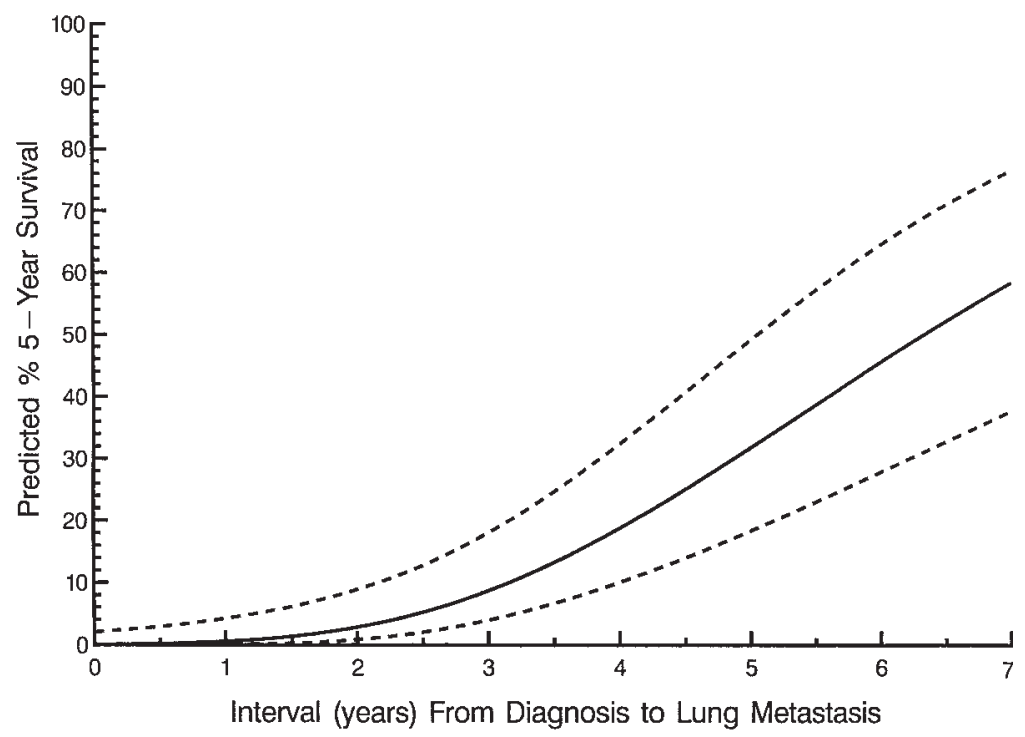

Fig 5. Predicted 5-year survival after the resection of the last metastasis, according to the interval between the colorectal cancer diagnosis and the resection and the appearance of the lung metastasis. The survival estimates (solid line) are enclosed within confidence limits (dashed line) equivalent to $1 \mathrm{SE}$.

Table III. Comparison of resection and nonresection patients

\begin{tabular}{|c|c|c|c|c|c|}
\hline \multirow[b]{2}{*}{ Variable/responses } & \multicolumn{2}{|c|}{ Resection (25) } & \multicolumn{2}{|c|}{ Nonresection (23) } & \multirow[b]{2}{*}{$\mathrm{P}$ value* } \\
\hline & $n$ & $\%$ & $n$ & $\%$ & \\
\hline \multicolumn{6}{|l|}{ Demographic data } \\
\hline \multicolumn{6}{|l|}{ Gender } \\
\hline Female & 16 & 64 & 8 & 35 & .04 \\
\hline Male & 9 & 36 & 15 & 65 & \\
\hline \multicolumn{6}{|l|}{ Colorectal cancer } \\
\hline \multicolumn{6}{|l|}{ TNM stage } \\
\hline I & 1 & 4 & 0 & 0 & $<.001$ \\
\hline II & 6 & 24 & 1 & 4 & \\
\hline III & 10 & 40 & 1 & 4 & \\
\hline IV & 8 & 32 & 21 & 91 & \\
\hline \multicolumn{6}{|c|}{ Type of colorectal cancer } \\
\hline Left & 22 & 88 & 11 & 48 & .003 \\
\hline Right & 3 & 12 & 12 & 52 & \\
\hline \multicolumn{6}{|c|}{ Chemotherapy after colorectal operation } \\
\hline Yes & 13 & 52 & 9 & 39 & .4 \\
\hline No & 12 & 48 & 14 & 61 & \\
\hline \multicolumn{6}{|l|}{ Liver metastases } \\
\hline \multicolumn{6}{|c|}{ Liver metastases (No.) } \\
\hline Single & 15 & 60 & 3 & 13 & $<.001$ \\
\hline Multiple & 10 & 40 & 20 & 87 & \\
\hline \multicolumn{6}{|l|}{ Lung metastases } \\
\hline Unilateral & 17 & 68 & 6 & 26 & .004 \\
\hline Bilateral & 8 & 32 & 17 & 74 & \\
\hline \multicolumn{6}{|c|}{ Lung metastases (No.) } \\
\hline Single & 10 & 40 & 3 & 13 & .036 \\
\hline Multiple & 15 & 60 & 20 & 87 & \\
\hline
\end{tabular}

$* \chi^{2}$ test. 


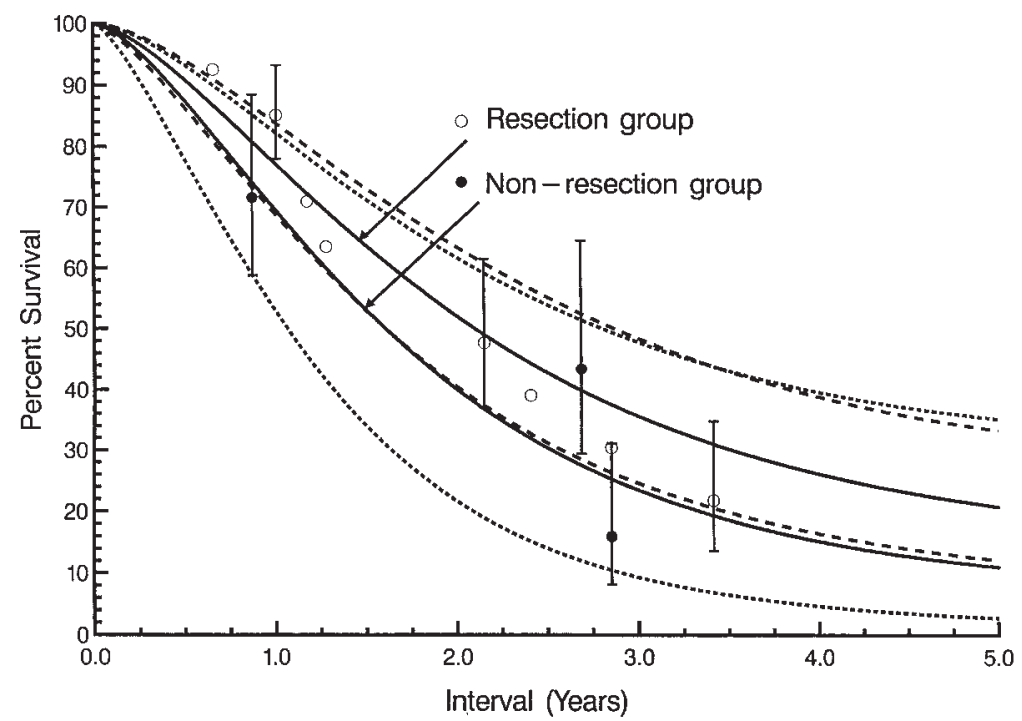

Fig 6. Survival after the appearance or resection of the metastasis in patients with a solitary liver metastasis, stratified according to resection and nonresection. (See Fig 1 for further explanation.)

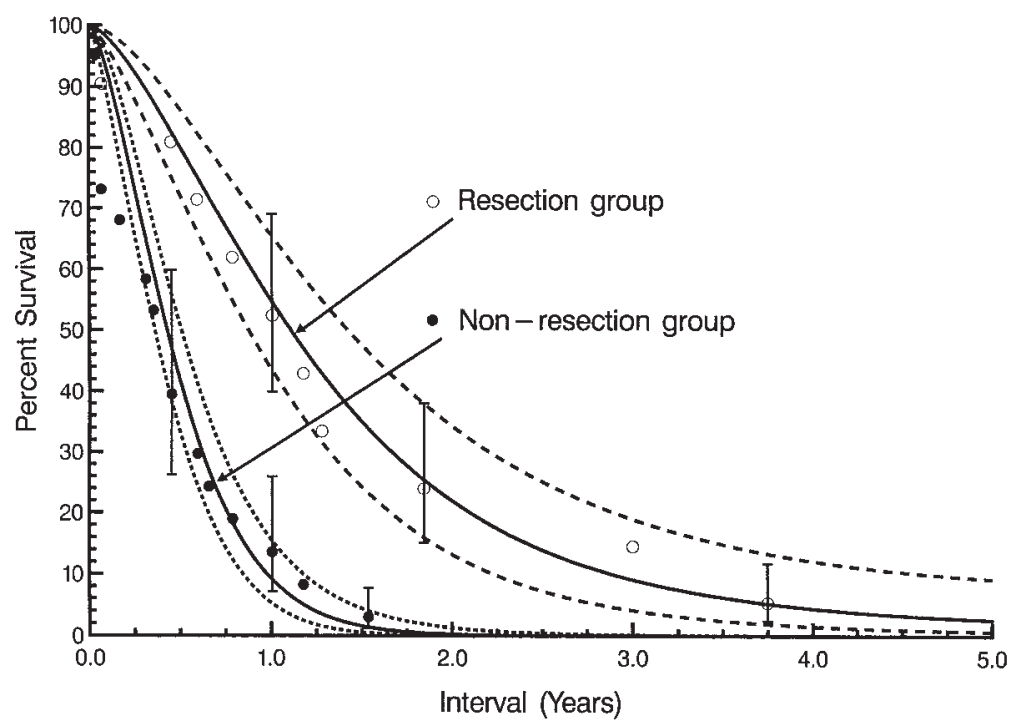

Fig 7. Survival after the appearance or resection of metastasis in patients who had multiple liver metastases, stratified according to resection and nonresection. (See Fig 1 for further explanation.)

life-table analyses, and decile risk analysis of ordinal and continuous variables to determine possible transformations of scale. A directed technique of stepwise entry of variables into the multivariable risk factor model was then used. ${ }^{10}$ The $P$ value criterion for retention of variables in the models was .1. Separate analyses were performed for the resection patients, the nonresection patients, and the combined group, which included group interaction terms. Regression coefficients are presented \pm 1 SE.
SPECIFIC TYPES OF MULTIVARIABLE ANALYSES. Three types of multivariable analyses were conducted to identify risk factors for death: (1) Cox semiparametric proportional hazards regression, ${ }^{11}(2)$ parametric hazard function regression, ${ }^{9}$ and (3) bootstrap hazard function regression. The bootstrap hazard function regression was used to verify the other multivariable analyses; in this analysis, automated forward riskfactor selection was performed on 1000 data sets, each consisting of a 5-time resampling of the original data set. 
Table IV. Relationship of individual variables to death (Cox proportional hazards method)

\begin{tabular}{|c|c|c|c|}
\hline Variable/responses & $\mathrm{P}$ value & Hazard ratio & $\begin{array}{c}\text { 95\% Confidence } \\
\text { limit }\end{array}$ \\
\hline Nonresection vs resection & $<.001$ & 3.40 & $1.78-6.50$ \\
\hline Male vs female & .2 & 1.52 & $0.83-2.78$ \\
\hline Age (y) at colorectal operation (per 10-y increase) & .02 & 1.52 & $1.08-2.13$ \\
\hline \multicolumn{4}{|l|}{ Colorectal cancer } \\
\hline \multicolumn{4}{|l|}{ TNM stage } \\
\hline III vs I-II & .4 & 0.67 & $0.25-1.81$ \\
\hline IV vs I-II & .5 & 1.33 & $0.60-2.94$ \\
\hline Type of colorectal cancer (left vs right) & 6 & 0.85 & $0.44-1.63$ \\
\hline Chemotherapy after colorectal operation (yes vs no) & .7 & 0.90 & $0.49-1.66$ \\
\hline \multicolumn{4}{|l|}{ Timing of colon cancer and metastatectomy } \\
\hline Mixed/synchronous vs metachronous & $<.001$ & 3.33 & $1.64-6.75$ \\
\hline Mixed vs metachronous & .1 & 2.35 & $0.94-5.88$ \\
\hline Synchronous vs metachronous & $<.001$ & 4.01 & $1.89-8.50$ \\
\hline \multicolumn{4}{|l|}{ Liver metastases } \\
\hline No. of liver metastases (multiple vs single) & $<.001$ & 3.37 & $1.72-6.61$ \\
\hline Interval from colorectal cancer to liver cancer (per 1-mo increase) & .02 & 0.97 & $0.95-0.99$ \\
\hline \multicolumn{4}{|l|}{ Lung metastases } \\
\hline Bilateral vs unilateral & .7 & 1.15 & $0.63-2.11$ \\
\hline No. of lung metastases (multiple vs single) & .1 & 1.77 & $0.87-3.60$ \\
\hline Interval from colorectal cancer to lung cancer (per 1-mo increase) & $<.001$ & 0.97 & $0.95-0.98$ \\
\hline
\end{tabular}

Table V. Risk factors for death after the appearance of both hepatic and pulmonary metastases (Cox proportional hazards method; $n=48$ )

\begin{tabular}{|c|c|c|c|}
\hline Variable/responses & $\mathrm{P}$ value & Hazard ratio & $\begin{array}{c}\text { 95\% Confidence } \\
\text { limit }\end{array}$ \\
\hline \multicolumn{4}{|l|}{ Model 1} \\
\hline Age (y) at colorectal operation (per 10-y increase) & .028 & 1.48 & $1.05-2.11$ \\
\hline No. of liver metastases (multiple vs single) & $<.001$ & 3.47 & $1.67-7.20$ \\
\hline Group (nonresection vs resection) & .013 & 2.47 & $1.21-5.03$ \\
\hline \multicolumn{4}{|l|}{ Model 2} \\
\hline Age (y) at colorectal operation (per 10-y increase) & .021 & 1.54 & $1.07-2.22$ \\
\hline No. of liver metastases (multiple vs single) & .006 & 2.74 & $1.33-5.63$ \\
\hline Interval from colorectal cancer to lung cancer (per 1-mo increase) & .011 & 0.97 & $0.96-0.99$ \\
\hline \multicolumn{4}{|l|}{ Model 3} \\
\hline Age (y) at colorectal operation (per 10-y increase) & .013 & 1.56 & $1.10-2.22$ \\
\hline No. of liver metastases (multiple vs single) & .001 & 3.27 & $1.59-6.74$ \\
\hline Timing of metastases (synchronous/mixed vs metachronous) & .031 & 2.27 & $1.08-4.79$ \\
\hline
\end{tabular}

These were summarized in terms of the frequency of selection of risk factors. ${ }^{12}$

NATURE AND INFLUENCE OF RISK FACTORS. Exploration of the influence of risk factors in the parametric multivariable analysis was performed by constructing a nomogram that represented the solution of the parametric equation for specific supplied values of each factor.

\section{Results}

\section{Benefit of metastasectomy}

Survival. Survival was longer in the resection group than in the nonresection group, whether calculated from first colorectal resection (first resection) or last appearance or resection of metastases (last metastasis). Median survival after the first colorectal cancer resection was 47 months in the resection group and 7 months in the nonresection group; 5 -year survival was $43 \%$ and $0 \%(P<.001)$. Median survival after the last appearance of metastases was 16 months in the resection group and 6 months in the nonresection group; 5-year survival was $9 \%$ and $0 \%(P<.001$; Fig 1$)$.

Time-related risk. When assessed from the time of last appearance of metastases, time-related instantaneous risk of death (hazard function) for resection and 


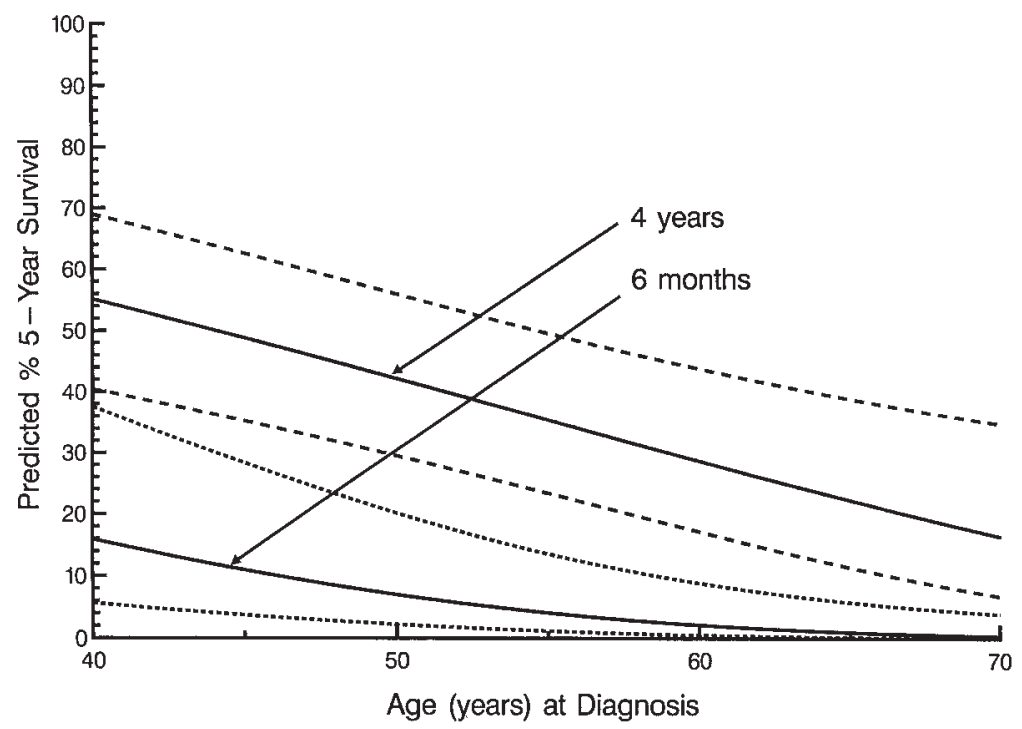

Fig 8. Predicted 5-year survival after the resection of the last metastasis, according to age at colorectal cancer diagnosis and resection. Two sets of estimates are shown that are solutions to the multivariable equation for death. The first is for a patient with a long (4 years) disease-free interval (from colorectal cancer to resection of pulmonary metastases) and the second is for a patient with a short (6 months) interval.

nonresection groups differed (Fig 2). For the resection group, the risk of death increased from last resection, peaked at 2 years, and declined thereafter. In contrast, the nonresection group's risk of death was both constant across time and systematically higher (nonoverlapping confidence limits; $P<.001$ ).

Timing and sequence of metastases. The timing and sequence of hepatic and pulmonary metastases differed in the resection and nonresection groups (Tables I and II). Synchronous disease was present at diagnosis in only 2 patients in the resection group and in all but 2 patients in the nonresection group.

In the resection group, patients with metachronous resections survived longer (median survival, 70 months) than patients with synchronous resections (median survival, 22 months) or those patients with mixed resections (median survival, 31 months) when calculated from first cancer resection $(P<.001)$.

However, in this group, survival was similar when calculated from the time of last metastatic resection; median survival was 22 months for metachronous resections, 14 months for synchronous resections, and 12 months for mixed resections $(P=.21$; Fig 3$)$. When synchronous and mixed resection groups were combined, survival was also similar; median survival was 22 months for metachronous resections and 14 months for combined resections $(P=.09$; Fig 4$)$.

The favorable influence of metachronous appearance
Table VI. Risk factors for death after the appearance of both hepatic and pulmonary metastases (parametric hazard function method; $n=48$ )

\begin{tabular}{lcl}
\hline Risk factors for death & Coefficient $\pm S D$ & P value \\
\hline Model $1 *$ & & \\
Longer interval to development & $-0.28 \pm 0.124$ & .02 \\
$\quad$ of pulmonary metastases (y) & $1.58 \pm 0.45$ & .0004 \\
Multiple hepatic metastases & $-0.97 \pm 0.45$ & .03 \\
Resection group & 1.93 & \\
Intercept & & \\
Model 2 $\dagger$ & & .01 \\
Longer interval to development & $-0.32 \pm 0.124$ & .002 \\
$\quad$ of pulmonary metastases $(y)$ & $1.13 \pm 0.37$ & .04 \\
Multiple hepatic metastases & $0.037 \pm 0.0182$ & \\
Older age at diagnosis & 0.154 & \\
Intercept & & \\
\hline
\end{tabular}

*Shaping parameter estimates: $\delta=0 ; \rho=5.73 ; \mathrm{v}=-0.670 ; \mathrm{m}=0$. $\dagger$ Shaping parameter estimates: $\delta=0 ; \rho=7.64 ; \mathrm{v}=-0.704 ; \mathrm{m}=0$.

of metastases was confirmed by multivariable analyses of death after the last metastasis resection. The only correlate found was the favorable influence of a longer interval between the colorectal cancer diagnosis and the development of pulmonary metastases $(P=.005)$. The influence and shape of a longer interval on 5-year survival is shown in Fig 5.

The number of patients with metachronous lesions in the nonresection group was too small to make mean- 


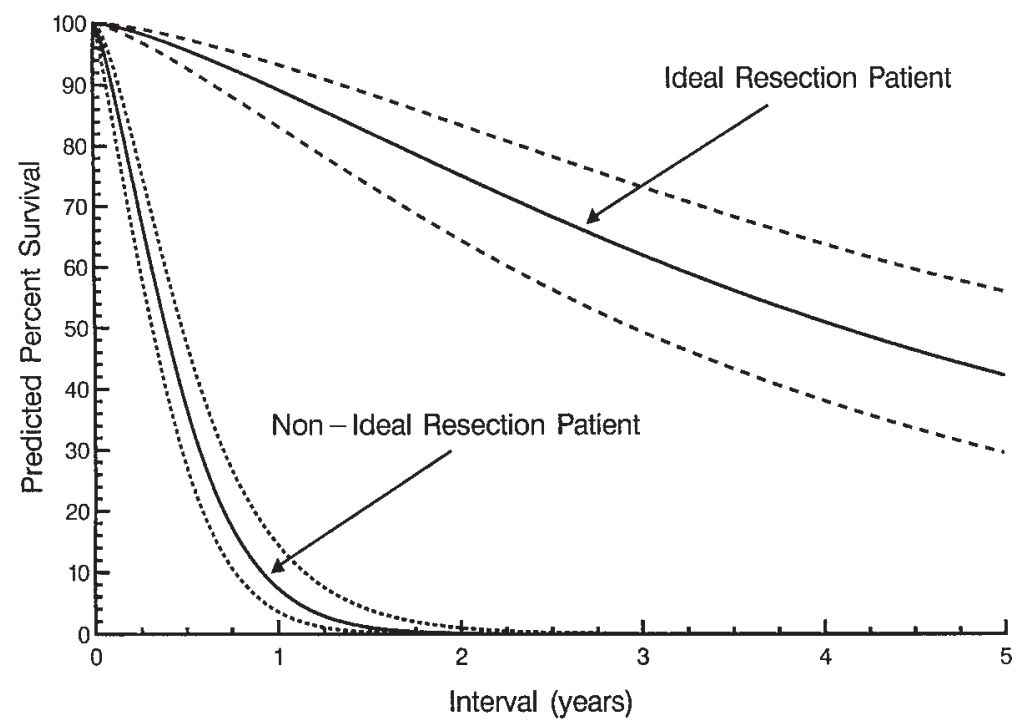

Fig 9. Survival after the resection of the last metastasis in a patient with ideal characteristics for resection (age, 50 years; solitary liver metastasis; 4-year interval from colorectal cancer resection to pulmonary metastasis), contrasted with a patient with nonideal characteristics for resection (age, 70 years; multiple liver metastases; synchronous disease).

ingful conclusions about timing and sequence of metastases.

The ideal patient for metastasectomy. Patients undergoing resections for liver and lung metastases were younger than those not undergoing resection. Age at the diagnosis of colorectal cancer in the resection group was $56 \pm 10$ years (median, 57 years; range, 37-72 years) and $61 \pm 8$ years in the nonresection group (median, 62 years; range, 46-77 years; $P=.04$ ). Patients who underwent resection were more likely to be women, to have a less advanced and left-sided colorectal cancer at the time of diagnosis, and to have solitary liver and lung metastases (Table III).

In the nonresection group, the 3 patients with solitary liver metastases fared equally well as their counterparts in the resection group (Fig 6).

The presence of multiple liver metastases was the only risk factor identified for death in the nonresection group $(P=.02)$. Survival was poor in patients with multiple liver metastases, but better in the resection group (Fig 7).

A number of variables were correlates of either poorer or longer survival from the appearance of the last metastasis, and many variables were correlated one with another (Table IV). Thus the multivariable analysis of the combined groups produced more than one equivalent model by all 3 methods of analyses, all consistent with the data (Tables V and VI). These analyses identified the younger patients at diagnosis of colorectal cancer (Fig 8), with a solitary liver metastasis, metachronous disease with a long disease-free interval, and resection of both liver and lung metastases as those more likely to enjoy long-term survival and possibly cure (Fig 9).

\section{Discussion}

Benefit of metastasectomy. More than $50 \%$ of patients undergoing curative resection of a colorectal carcinoma will experience recurrent disease. Liver and lung are the most common sites of distant metastases. On occasion, a patient will have metastases limited to the liver and lung after a curative colon resection. Inherently, these patients represent a highly select group. Does resection of metastases improve survival in these patients or is this a group of patients who will have a good survival regardless of treatment? ${ }^{13}$ The rarity of these patients and the ethics of withholding resection in an ideal surgical candidate preclude a phase III study comparing surgical to nonsurgical care. This study was developed to illuminate the controversy of resection of liver and lung metastases after colorectal resection. It can be debated that the 2 groups of patients are not comparable. However, the important differences observed between the resection and nonresection groups provide valuable insight into the selection process. 
The benefit of resection can be best understood by the evaluation of the hazard functions of the 2 groups. For the nonresection group, the risk of death is constant. In contrast, the biphasic hazard function of the resection group illustrates the benefit of resection. If the resection group was comprised of a poor-risk group who rapidly die of the disease and a good-risk group who have prolonged survival despite treatment, the hazard function would be that of an exponential decay from the risk of nonresection group to the risk of the resection group. However, for the resection group, the risk of death after resection slowly rises to a peak at 2 years. Surprisingly, the risk falls to a level similar to that of early postoperative risk and is persuasive evidence that, despite selection, resection provides a survival advantage.

Timing and sequence of metastases. The timing of metastases greatly influences survival. Metachronous metastases provide an intrinsic survival advantage. These patients must survive the resection of the colorectal primary and, after a disease-free interval, undergo resection of the first metastatic site. After a second disease free-interval, metastasectomy is performed at the second site. If survival is calculated from the colorectal resection or the first metastasectomy, a patient with metachronous metastases will almost always have better survival. However, if the date of last resection (the time at which the patient is rendered cancer free) is chosen for the calculation of survival, the timing of metastases is no longer a factor that influences survival.

The sequence of metastases also influenced survival. Most patients with metachronous or mixed disease had a sequence of metastases to the liver and then the lung. Patients with synchronous disease or metastases to the lung and then the liver had a poor survival. This implies that the route of metastases is crucial for patients to benefit from resection of both liver and lung metastases. Patients with cascade metastases from the colorectal primary through the portal venous system to the liver and then through the systemic venous system to the lung are most likely to have a metachronous pattern of metastases. Patients with simultaneous metastases via the portal venous system to the liver and the systemic venous system to the lung have a reduced survival.

The ideal patient for metastasectomy. Selection of patients for resection of hepatic and pulmonary metastases is an important factor that determines survival. Unfortunately, the tumor and host factors that allow local resection to control systemic disease are unknown. Multivariable analyses were used to adjust for important differences between and within the groups. Although not an adequate substitute for a randomized trial, it clar- ifies which patients are more likely to benefit from resection. Despite the limitations of a small study group, valuable information has been learned to assist in the selection and management of these patients.

\section{Conclusions}

In select patients with colorectal cancer, resection of metachronous solitary liver and lung metastases improves survival and may result in cure. Resection of multiple synchronous metastases may provide longer palliation than conventional therapy.

\section{REFERENCES}

1. Goya T, Miyazawa N, Kondo H, Tsuchiya R, Naruke T, Suemasu K. Surgical resection of pulmonary metastases from colorectal cancer: 10-year follow-up. Cancer 1989;64:1418-21.

2. Griffith KD, Sugarbaker PH, Chang AE. Repeat hepatic resections for colorectal metastases. Surgery 1990;107:101-4.

3. Smith JW, Fortner JG, Burt M. Resection of hepatic and pulmonary metastases from colorectal cancer. Surg Oncol 1992;1: 399-404.

4. Yano T, Hara N, Ichinose Y, Yokoyama H, Miura T, Ohta M. Results of pulmonary resection of metastatic colorectal cancer and its application. J Thorac Cardiovasc Surg 1993;106:875-9.

5. Gough DB, Donohue JH, Trastek VA, Nagorney DM. Resection of hepatic and pulmonary metastases in patients with colorectal cancer. Br J Surg 1994;81:94-6.

6. Girard P, Ducreux M, Baldeyrou P, Rougier P, Chevalier TL, Bougaran J, et al. Surgery for lung metastases from colorectal cancer: analysis of prognostic factors. J Clin Oncol 1996;14: 2047-53.

7. Ambiru S, Miyazaki M, Ito H, Nakagawa K, Shimizu H, Kato A, et al. Resection of hepatic and pulmonary metastases in patients with colorectal carcinoma. Cancer 1998;82:274-8.

8. Kaplan EL, Meier P. Nonparametric estimation from incomplete observations. J Am Stat Assoc 1958;53:457-81.

9. Blackstone EH, Naftel DC, Turner ME Jr. The decomposition of time-varying hazard into phases, each incorporating a separate stream of concomitant information. J Am Stat Assoc 1986; 81:615-24.

10. Baskerville JC, Toogood JH. Guided regression modeling for prediction and exploration of structure with many explanatory variables. Technometrics 1982;24:9-17.

11. Cox DR. Regression models and life tables. J R Stat Soc B 1972; $34: 187-220$

12. Altman DG, Anderson PK. Bootstrap investigation of stability of a Cox regression model. Stat Med 1989;8:771-83.

13. Äberg T. Selection mechanisms as major determinants of survival after pulmonary metastasectomy. Ann Thorac Surg 1997;63:611-2.

\section{Discussion}

Dr Mark S. Allen (Rochester, Minn). Since Alfred Blalock first reported on pulmonary metastasectomy for colorectal metastases in 1944, many centers, including our own, have reported their results. Unfortunately, all of these results, as is the current one, are retrospective. They are all unable to answer the question that a randomized trial could: Is it beneficial to resect metastatic disease? 
We in the surgical community have come to accept metastectomy as a beneficial technique, but there are those in the medical field who still question the efficacy of this procedure. Ethically, it is nearly impossible to carry out such a prospective trial. Thus we are left with retrospective reviews, such as this one. This does not mean that these reviews are not useful. Quite the contrary. When properly done, they can provide us with information about factors that lead to improved survival in selected groups of patients.

Several authors have reported a number of factors, including age, disease-free interval, number of metastases, and low carcinogenic embryonic antigen level, which impart and improve survival. In this article, the authors have performed a careful and complete statistical analysis of a relatively small group of patients who have undergone resection of both hepatic and pulmonary metastases. They attempt to draw a conclusion between a group of patients who underwent resection and a group who did not undergo resection.

I would caution the audience that this comparison has limited validity because there is an obvious bias of selection in favor of the patients having resection. The authors have shown through multivariate analysis that patients who are young, who have solitary hepatic metastases, who later experience pulmonary metastases, and who have a long diseasefree interval survive longer.

First, in our paper and in others, patients with an elevated carcinoembryonic antigen level had a worse 5-year survival than those with a normal level. Did you observe this finding? If so, what do you think the significance of it is?

Second, what do you use as a criteria to turn down patients for resection? Is there a number of nodules that is too many to have a resection? How do you select patients for an operation, and how do you decide which ones will not undergo an operation?

Third, from a technical standpoint, do you perform the operation as a combined hepatic and pulmonary resection or do you stage it?

Dr Robinson. Thank you for your questions. Carcinoembryonic antigen levels were not consistently measured in these patients. Because this information was incomplete, it was not included in the analysis.

There was no protocol for patient selection. Only clinical evaluation and the surgeon's judgment were used to select patients for resection. There was no cutoff for the number of metastases. Indeed, one patient had 7 bilateral pulmonary metastases resected.

Only 2 patients had synchronous resections, although 23 patients had metachronous resections. Our experience is with staged resections. This approach allows operative confirmation that the first metastatic site can be resected and that there are no unexpected metastases in the operative field. Staging of the resections allows recovery time and a reassessment period to assure there is no progression of metastatic disease or recurrence at previous resection sites.

If the resections are to be synchronous, it is best to complete one resection before embarking on the second. Doing the hepatic resection first allows assessment of the primary colorectal resection site and the abdominal cavity, common sites of recurrence.

Dr Larry S. Kaiser (Philadelphia, Pa). Getting back to some of the selection factors when you went back and looked at these charts, was age a major criteria in selecting the patients as best as you can tell retrospectively? Can you comment on how many of these patients would have received 5-fluorouracil, which is the standard agent for the treatment of colorectal cancer and is used as an adjuvant? In light of the fact that data will be presented at the American Society of Clinical Oncology meeting that shows that CPT-11, now called irinotecan, has been shown to be efficacious in patients whose condition did not respond to 5-fluorouracil, how would that knowledge (that is, a new drug effective in a solid tumor like colon cancer) have an impact on these types of operations?

Dr Robinson. It did not appear from the retrospective analysis that age was a criterion for metastasectomy. If a patient was medically fit for resection, the patient was deemed a candidate for resection.

We did compare the survival of patients who received chemotherapy to the survival of those patients who did not. Equal numbers of patients in both groups received chemotherapy, 13 of 25 patients in the resection group and 9 of 23 patients in the nonresection group. Chemotherapy did not affect survival. The regimens were 5-fluorouracil based; no patient received irinotecan (CPT-11).

Dr John A. Odell (Jacksonville, Fla). Which one would you do first, the lung or the liver metastases? My approach is to do the liver first because there might be unsuspected peritoneal seedings or other spread that would prevent you from performing the thoracic part of the procedure. Have you ever found any of these situations where at the time of managing the liver metastases you found that it was not indicated to proceed with the pulmonary resection?

Dr Robinson. In the uncommon situation of synchronous metastases, we agree that it makes sense to do the abdominal exploration and hepatic resection first. This confirms that there are no unexpected local recurrences or abdominal metastases and that the hepatic metastases are resectable. 